J. Lake Sci.(湖泊科学), 2009, 21(2): 255-263

http://www.jlakes.org. E-mail: jlakes@niglas.ac.cn

(C)2009 by Journal of Lake Sciences

\title{
云南高原湖泊有色可溶性有机物和颗粒物光谱吸收特性*
}

\author{
张运林 ${ }^{1}$, 张恩楼 ${ }^{1}$, 刘明亮 ${ }^{2}$ \\ (1: 中国科学院南京地理与湖泊研究所湖泊与环境国家重点实验室太湖湖泊生态系统研究站, 南京 210008) \\ (2: 南京农业大学资源与环境科学学院, 南京 210095)
}

摘 要: 云南高原湖泊是我国湖泊分布最密集的五大湖群之一, 不但湖泊数量众多而且类型多样. 由于湖泊所处位置海拔较 高, 容易受日益增强 UV-B 辐射影响. 通过对云南高原 34 个湖泊有色可溶性有机物和颗粒物吸收测定, 分析其光谱吸收特性 及对总吸收的贡献, 有利于深刻理解紫外辐射在高原湖泊内衰减. 不同湖泊间 CDOM 吸收差异明显, 其大小与水体营养盐状 况相关, CDOM 吸收系数与水体总氮存在显著正相关. 增加背景项的指数函数模型能最好模拟 CDOM 光谱吸收. 除在浮游植 物浓度非常高的杞麓湖、听湖、星云湖, 颗粒物吸收系数在 $675 \mathrm{~nm}$ 附近存在一个吸收峰外, 其它湖泊总颗粒物光谱吸收大致 随波长的增加吸收系数逐渐降低, 呈现非色素颗粒物光谱吸收特征, 整体上颗粒物吸收以非色素颗粒物为主. CDOM 对总吸 收的贡献主要集中在 $600 \mathrm{~nm}$ 以下波长, 尤其是 $400 \mathrm{~nm}$ 以下的紫外波段, 其在紫外波段(350-400nm)的贡献明显要大于光合有 效辐射波段 $(400-700 \mathrm{~nm}$ )(ANOVA, $P<0.001$ ). 特别对于透明度 $\mathrm{SD} \geqslant 1.0$ 的清澈型湖泊, CDOM 吸收对紫外辐射衰减的贡献更大, 其吸收很大程度上决定了紫外辐射的影响深度.

关键词: 云南高原; UV-B 辐射; 吸收系数; 有色可溶性有机物; 悬浮物

\section{Spectral absorption properties of chromophoric dissolved organic matter and particulate matter in Yunnan Plateau lakes}

ZHANG Yunlin $^{1}$, ZHANG Enlou ${ }^{1} \&$ LIU Mingliang ${ }^{2}$

(1: Taihu Lake Laboratory Ecosystem Research Station, State Key Laboratory of Lake Science and Environment, Nanjing Institute of

Geography and Limnology, Chinese Academy of Sciences, Nanjing 210008, P.R.China)

(2: College of Natural Resources and Environmental Science, Nanjing Agricultural University, Nanjing 210095, P.R.China)

Abstract: The Yunnan Plateau lakes with different lake types, located in Yunnan Province in southwestern China, are one of the five densest lake areas in China. The lakes, which are generally distributed at elevations from $1200 \mathrm{~m}$ to $4000 \mathrm{~m}$ above sea level, receive high intensity UV-B radiation. In this study, the spectral absorption properties of chromophoric dissolved organic matter(CDOM) and particulate matter are studied based on a wide investigation including 34 lakes in the Yunnan Plateau. The study will be helpful in the understanding of the ultraviolet radiation attenuation and the effects of increased UV-B radiation on lake ecosystems. A wide range of variability in CDOM absorption was found among the Yunnan Plateau lakes. CDOM absorption coefficient generally corresponded to the nutrient level with a significantly positive linear correlation between $a_{\text {СDом}}(320)$ and total nitrogen concentration. Exponential model with constant parameters of background items could give the most approximate estimation of CDOM absorption spectra. A marked phytoplankton absorption peak around 675nm was only observed in Qiluhu, Tinghu and Xinyunhu with very high chlorophyll a concentration. High contribution of CDOM to the total absorption was found during the wavelength range lower than $600 \mathrm{~nm}$ especially in the ultraviolet range lower than $400 \mathrm{~nm}$. The contribution of CDOM to the total absorption was significantly higher in UVR range (350-400nm) than in PAR range (400-700nm) (ANOVA, $P<0.001)$. For the clear lake water with secchi disc: SD $\geqslant 1.0$, the contribution of CDOM absorption to the diffuse attenuation of ultraviolet radiation would increase, and CDOM absorption, with a large probability, would determine the attenuation depth of ultraviolet radiation.

* 国家自然科学基金项目(40601099, 40772204)和中国科学院知识创新工程方向性项目(KZCX2-YW-419)联合资助. 2008-05-12 收稿; 2008-08-26 收修改稿. 张运林, 男, 1976 年生, 博士, 副研究员; E-mail: ylzhang@niglas.ac.cn. 
Keywords: Yunnan Plateau lakes, UV-B radiation, absorption coefficient, chromophoric dissolved organic matter, suspended matter

人类活动产生的大量氯氟烃化合物和哈龙等卤族化合物等进人平流层后造成了臭氧层厚度减薄, 从 而使得到达地表的太阳紫外辐射尤其是对人体有害的 UV-B 辐射(280-320nm)明显增加. 臭氧层厚度每减 少 $1 \%$, 到达地表的 UV-B 辐射增强 $2 \%$. 自 1980 年以来，春季时南极和北极 UV-B 辐射分别升高约 $130 \%$ 和 $22 \%$, 南半球和北半球中纬地区 UV-B 辐射也有不同程度升高 ${ }^{[1]} .20$ 世纪 90 年代初, 我国北京、昆明、 黑龙江、浙江、青海等地均观测到当地臭氧总量不断减少、研究显示, 每年 6 至 9 月, 在青藏高原 $\left(33.5^{\circ} \mathrm{N}\right.$, $\left.90.6^{\circ} \mathrm{E}\right)$ 上空存在一个大气臭氧低值中心, 中心臭氧柱总量月平均值比同纬度东部地区平均值低 $11 \%$ 左右. 而且平均年递减率超过 $0.3 \%$, 臭氧低值中心在逐年加深 ${ }^{[2]}$. 近 20 年来我国上空的臭氧含量均有不同程度 的减少, 面临 UV-B 辐射增强的严峻形势. 当前 UV-B 辐射增强对陆地和水生生态系统的影响成为国际上 关注的焦点和研究前沿.

许多研究表明, 湖泊中有色可溶性有机物(Chromophoric Dissolved Organic Matter, CDOM)或溶解性 有机碳(Dissolved Organic Carbon, DOC)浓度很大程度上控制着 UV-B 辐射在湖泊中的传输与衰减 ${ }^{[3-7]}$. 如 Scully 等 ${ }^{[5]}$ 对大量湖泊的 UV-B 辐射衰减系数与叶绿素 a、DOC 和颗粒有机碳浓度建立数值关系, 发现 UV-B 辐射衰减系数与 DOC 浓度相关性最显著. 但近年来也有一些研究发现 UV-B 衰减除受水体中 CDOM 的影响外, 还要受浮游植物和非藻类悬浮物的影响 ${ }^{[4,8-11]}$. Smith 等 ${ }^{[8]}$ 在对低浓度 DOC 的大型湖泊 伊利湖紫外辐射的研究中发现 UV-B 衰减系数有时与 DOC 浓度的相关性非常低, 决定系数仅 0.14. 总悬 浮物浓度是伊利湖预测 UV-B 和 UV-A(320-400nm)衰减的最有效因子, 与 UV-B、 UV-A 波段衰减系数线 性相关的决定系数分别为 $0.91 、 0.83$. Belzilel 等 ${ }^{[9]}$ 在琵芭湖的研究发现颗粒物吸收和散射对紫外辐射衰减 起到显著作用, 平均而言 $340 \mathrm{~nm}$ 处 CDOM 和颗粒物吸收对衰减的贡献率分别为 $66 \%$ 和 $21 \%$. 因此了解 $\mathrm{CDOM}$ 和颗粒物的吸收等光学特性对于预测紫外辐射在湖泊内衰减及对水生生物的影响至关重要. 本文 主要是通过对云南高原 34 个湖泊 CDOM 和颗粒物吸收测定, 探讨其光谱吸收特性及对总吸收的贡献, 便 于模拟紫外辐射在高原湖泊的衰减.

\section{1 材料与方法}

\section{1 研究区域}

云南高原湖泊是我国湖泊分布最密集的五大湖群之一 ${ }^{[12]}$. 由于海拔较高, 接受的 UV-B 辐射绝对量 较大, 一般海拔每增高 $1000 \mathrm{~m}$, UV-B 辐射增加 $10 \%-20 \%{ }^{[13]}$. 此外, 高原湖泊由于受人类活动相对较小, 溶解性物质浓度较低, UV-B 辐射穿透的深度较深, 一般达好几米甚至更深 $[5-6,14]$, 并且其他因素引起的 CDOM 浓度的轻微变化都会造成 UV-B 穿透深度的迅速增加 ${ }^{[15]}$, 因而高原湖泊更易受增强的 UV-B 辐射 影响. 在云南高原湖泊开展 UV-B 辐射衰减机理及其生态效应的研究具有重要的现实意义. 除了 UV-B 辐 射较强外, 云南高原上湖泊海拔高度从 $1200 \mathrm{~m}$ 到 $4000 \mathrm{~m}$ 不等, 湖泊营养类型和生态类型多样, 既有星云 湖等典型的富营养化浅水湖泊又有抚仙湖等典型的贫营养深水湖泊 ${ }^{[12]}$.

2005年6-7月在云南高原总共调查了 38 个湖泊, 海拔高度从 $1291 \mathrm{~m}$ 到3809m不等, 包括不同营养类型 的湖泊, 这次调查几乎覆盖了云南高原所有面积大于 $1 \mathrm{~km}^{2}$ 湖泊. 由于本项研究主要是探讨不同湖泊受紫 外辐射的可能影响, 因而每个湖泊只布设了一个采样点, 采样点一般选择在湖中心位置, 取水体表层样. 38 个湖泊中只有 34 个湖泊进行了颗粒物吸收的测定 (未包括芘碧湖、抚仙湖、海子边、圆湖), 为便于分析, 本文研究针对这34个湖泊, 空间分布见图1, 各湖泊位置等基本状况参见文献[16].

\section{$1.2 \mathrm{CDOM}$ 和颗粒物吸收测量}

CDOM 的光谱吸收系数测定详细描述参见文献 ${ }^{[17]}$. 由于高原湖泊 CDOM 吸收较小, 在 $650 \mathrm{~nm}$ 以上 波段吸光度噪声比较大, 这里选择 650-700nm 的平均值进行散射效应订正.

$$
a_{\mathrm{CDOM}}(\lambda)=a_{\mathrm{CDOM}}\left(\lambda^{\prime}\right)-a_{\mathrm{CDOM}}(\text { mean }) \lambda / 675
$$

式中: $a(\lambda)$ 为波长 $\lambda$ 的吸收系数 $\left(\mathrm{m}^{-1}\right) ; a_{\mathrm{CDOM}}\left(\lambda^{\prime}\right)$ 为波长 $\lambda$ 的未校正吸收系数 $\left(\mathrm{m}^{-1}\right) ; a_{\mathrm{CDOM}}($ mean $)$ 为 $650-700 \mathrm{~nm}$ 
处 CDOM 吸收系数的平均值; $\lambda$ 为波长 $(\mathrm{nm})$.

非色素颗粒物的吸收系数采用定量滤膜技术测定, 具体操作与计算参见文献[17]. 总吸收为 CDOM、 颗粒物和纯水吸收的线性加和.

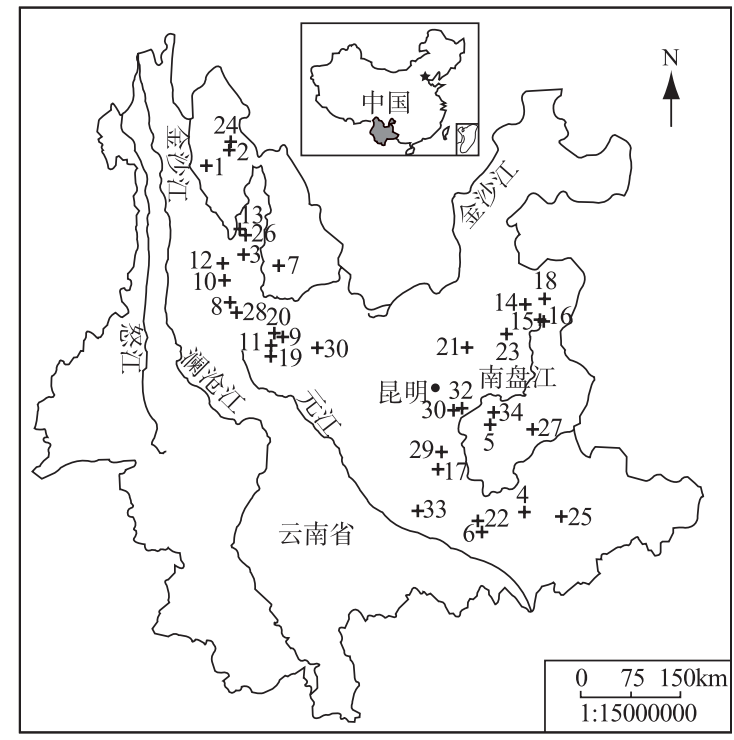

1 碧沽天池 2 碧塔海 3 草海 4 差 黑海 5 长湖 6 长桥海 7 程海 8 洱 海 9 海哨水库 10 海西海 11 浑水 海 12 剑湖 13 拉什海 14 李家凹 15 马塘水库 16 平地海 17 杞麓湖 18 前屯水库 19 青海 20 清涧美 21 清水海 22 三角海 23 石坡海 24 属 都湖 25 听湖 26 文笔海 27 无良海 28 西湖 29 星云湖 30 阳宗海 31 羊派水库 32 宜良草海 33 异龙湖 34 月湖

图 1 调查湖泊的空间分布

Fig.1 Distribution of sampling lakes in Yunnan Plateau

\section{3 其他参数测定}

透明度用 $30 \mathrm{~cm}$ 的黑白赛氏盘测定. 总悬浮物烘干称重法测定. 叶绿素 $\mathrm{a}$ 的测定采用分光光度法, 用 GF/C 滤膜过滤水样, 然后将滤膜置于冰箱中冷冻 $48 \mathrm{~h}$ 以上, 取出用 $90 \%$ 的热乙醇萃取, 然后在岛津 UV2401 分光光度计上测定 750、663、645、630nm 处吸光度，换算得到叶绿素 a. 总氮、总磷测定用碱 性过硫酸钾消解, 紫外分光光度法测定总氮, 钿酸铵分光光度法测定总磷.

\subsection{CDOM 光谱吸收斜率计算}

尽管 CDOM 吸收被广泛认为随波长增加近似指数函数衰减, 即可以用式(2)进行模拟，但近年来也有 研究表明简单指数模型并不是模拟 CDOM 光谱吸收的最佳模型 ${ }^{[18-19]}$. 如一些学者对指数模型进行改进, 在(2)式右端增加一个背景项参数 $K^{[18-19]}$. 这里我们选取常用的 3 种指数模型模拟 CDOM 光谱吸收(表 1), 拟合波段范围为 $280-500 \mathrm{~nm}$.

$$
a_{\mathrm{CDOM}}(\lambda)=a_{\mathrm{CDOM}}(440) \exp [S(440-\lambda)]
$$

表 $1 \mathrm{CDOM}$ 光谱吸收系数模型

Tab.1 The three models considered as descriptors of CDOM absorption spectra

\begin{tabular}{llll}
\hline 模型描述 & 函数表达式 & 模型参数 & 拟合方法 \\
\hline 模型 1: 吸收系数取自然对数线性拟合指数模型 & $\ln a_{\mathrm{CDOM}}(\lambda)=a_{1}-S_{1} \lambda$ & $a_{1}, S_{1}$ & 线性拟合 \\
模型 2: 非线性拟合指数模型 & $a_{\mathrm{CDOM}}(\lambda)=a_{2} \mathrm{e}^{S_{2}(440-\lambda)}$ & $a_{2}, S_{2}$ & 非线性拟合 \\
模型 3: 增加背景项指数模型 & $a_{\mathrm{CDOM}}(\lambda)=a_{3} \mathrm{e}^{S_{3}(440-\lambda)}+K$ & $a_{3}, S_{3}, K$ & 非线性拟合 \\
\hline
\end{tabular}




\section{5 数据统计分析}

运用 SPSS11.0 进行数据统计分析, 包括计算平均值、标准差、方差分析、线性回归分析等. CDOM 光谱斜率的计算在 Matlab 软件支持下完成, 利用 Matlab 里面的优化工具箱(Optimization Toolbox), 采用 最小二乘法计算得到. 引人决定系数 $\left(R^{2}\right) 、$ 均方根误差(Root mean square error, RMSE)来检验模拟值和实测 值是否一致的评判标准. RMSE 计算式如下:

$$
R M S E=\sqrt{\frac{\sum_{i=1}^{n}\left(x_{\mathrm{Ess}, i}-x_{\mathrm{Obs}, i}\right)^{2}}{n}}
$$

式中, $x_{\mathrm{Est}, i}, x_{\mathrm{Obs}, i}$ 分别为第 $\mathrm{i}$ 波长处模拟值和实测值, $n$ 为 $400-700 \mathrm{~nm}$ 波长总数.

\section{2 结果与讨论}

\subsection{CDOM吸收}

$\mathrm{CDOM}$ 的光谱吸收曲线随波长增加逐渐递减, 到长波的 $650 \mathrm{~nm}$ 以上趋向于零, 所有站点都一样看不 出差别, 但越到短波其差异越显著(图2). $a_{\mathrm{CDOM}}(320)$ 的变化范围为 $0.65-14.05 \mathrm{~m}^{-1}$, 均值为 $4.83 \pm 3.85 \mathrm{~m}^{-1}$, 不同湖泊间差异非常明显, 最高值为最低值的21.6倍(表2). 与世界上同海拔高度的阿尔卑斯上湖泊相比 ${ }^{[4,14]}$, 云南高原湖泊水体中CDOM浓度明显偏高, 这可能与云南高原四季如春的气候以及一些湖泊流域 高密度的人口和人类活动有关. 与太湖等长江中下游富营养浅水湖泊相比 ${ }^{[17,20]}$, 云南高原湖泊CDOM吸 收系数要小得多, 这主要是由于长江中下游浅水湖泊地处长江洪泛平原, 累积了大量溶解性物质, 加之 高强度的工农业生产向这些湖泊注人了高浓度的溶解性物质. 云南高原湖泊CDOM吸收大小与水体营养 盐状况相关, 一般营养水平高的湖泊, CDOM吸收系数也比较大, $a_{\mathrm{CDOM}}(320)$ 与水体总氮存在显著正相关 $\left(R^{2}=0.24, P<0.005\right)$. CDOM吸收与营养盐的这种正相关其实也不难理解, CDOM本身是一类溶解性有机 物, 自然包含了溶解性氮、磷营养盐. 此外, CDOM的来源不外乎流域外源输人和湖泊内浮游植物死亡降 解释放, 高浓度的氮磷营养盐一般都来自湖泊集水流域的输人, 因而自然也表示CDOM的外源输人比较 高, 而湖泊内高浓度的营养盐也有利于浮游植物的大量生长, 其死亡降解释放也增加了水体内CDOM浓 度 ${ }^{[4,21]}$. 当然, 对于接受紫外辐射较强的高原湖泊来说, 紫外辐射对CDOM的光化学降解也会影响到其生 物地球化学循环过程 ${ }^{[4,7,21]}$. 如Hayakawa等 ${ }^{[20]}$ 对抚仙湖水样进行光漂白实验发现, 当水样在太阳辐射下照 射数天后其DOC浓度降低了 $7 \%$.
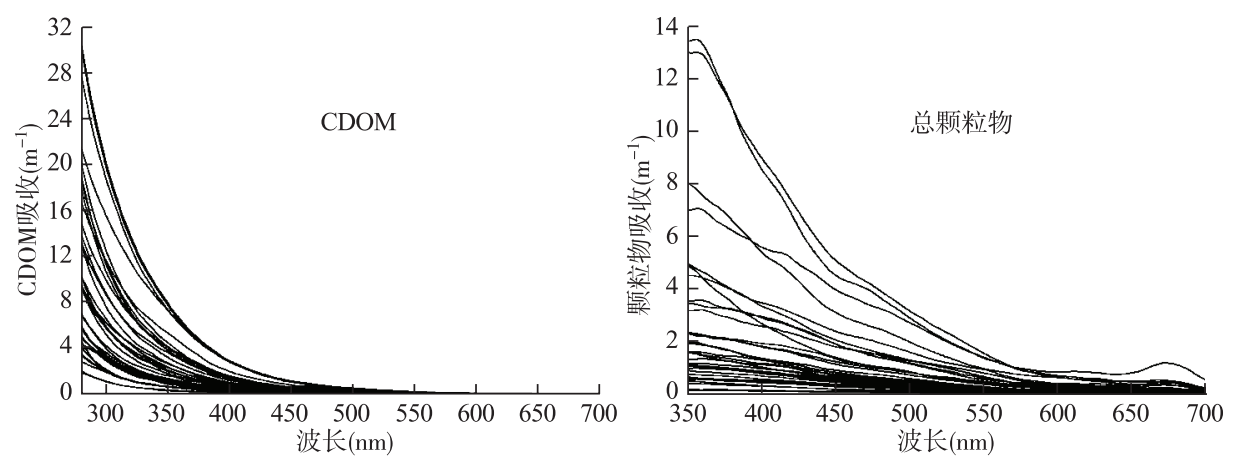

图 2 CDOM 和颗粒物吸收光谱

Fig.2 Absorption spectra of CDOM and total particulate matter

3 种模型计算得到的 CDOM 光谱斜率对比见表 2、3. 增加背景项后的指数函数模型模拟的结果最好 ( $R^{2}$ 最大、RMSE 最小), 而模型 1 的拟合结果是最差的(表 3). 事实上, 模型 1 单就式(2)两边取自然对数进 行线性拟合和模型 2 用式(2)进行非线性拟合得到的结果差别非常大, 模型 2 的结果明显要好于模型 1 , 模 
表 $2 \mathrm{CDOM}$ 和颗粒物特征波长吸收系数、CDOM 光谱斜率及相关理化参数 ${ }^{*}$

Tab.2 Absorption coefficients of CDOM, particulate matter and CDOM spectral slope, and related to physicochemical parameters

\begin{tabular}{|c|c|c|c|c|c|c|c|c|c|c|c|c|c|}
\hline & \multicolumn{2}{|c|}{$\begin{array}{c}a_{\mathrm{CDOM}}(\lambda) \\
\left(\mathrm{m}^{-1}\right)\end{array}$} & \multicolumn{2}{|r|}{$\begin{array}{l}a_{\mathrm{p}}(\lambda) \\
\left(\mathrm{m}^{-1}\right)\end{array}$} & \multicolumn{3}{|c|}{ CDOM 光谱斜率 $\left(\mu \mathrm{m}^{-1}\right)$} & \multirow[t]{2}{*}{$\begin{array}{c}K \\
\left(\mathrm{~m}^{-1}\right)\end{array}$} & \multirow[t]{2}{*}{ Chl.a } & & \multirow[t]{2}{*}{ SD } & \multirow[t]{2}{*}{$\mathrm{TN}$} & \multirow[t]{2}{*}{$\mathrm{TP}$} \\
\hline & 20 & 355440 & 55 & $440 \quad 675$ & $S_{1}$ & $S_{2}$ & $S_{3}$ & & & & & & \\
\hline & 99 & 3.760 .76 & 0.50 & 10 & & & & & & 20 & 15 & 76 & .0055 \\
\hline & 2.76 & 570.34 & 17 & 3 & & & & 19 & & 40 & .5 & 46 & 188 \\
\hline & 4. & 7 & 99 & 660 & 1880 & & & 0 & 1.49 & 7 & 1.2 & 30 & 0087 \\
\hline e & 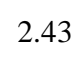 & 0 & & 780.36 & & & & & & 0 & 0.2 & 67 & 0190 \\
\hline & 1. & c & 75 & 12 & 25. & 502 & & 0.08 & 8 & .87 & .7 & 35 & 018 \\
\hline & 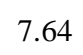 & 870.77 & - & (.) & & & & (2) & & 0.33 & 0.5 & .98 & . \\
\hline & 1. & 0.20 & 16 & 20 & & & & & & 30 & 6 & 51 & 0.0213 \\
\hline & 5 & 37 & 59 & 10 & 18 & & & 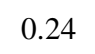 & & 3.30 & 1.3 & 59 & 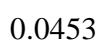 \\
\hline & + & & St & . & & & & & & 3.10 & 0.0 & (5) & $0<2$ \\
\hline & 1.23 & 660.14 & 64 & 2 & 20. & & & & & 2.70 & .2 & .21 & 268 \\
\hline & 1.80 & & 34 & 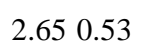 & & & & . & & 10 & 0.4 & 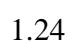 & $x$ \\
\hline & & & 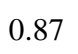 & 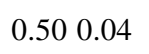 & & & & & 0.01 & 47 & 2 & 0 & 107 \\
\hline & 3.85 & & 26 & & & & & -0 & 2.20 & 32.40 & 0.7 & 0.67 & 0438 \\
\hline & 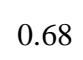 & & 7 & 090.01 & & & & & & 27 & 3.0 & 0.30 & \\
\hline & .21 & 30.15 & 2 & 0 & & & & & & 1.10 & .0 & 6 & 09 \\
\hline & 2.44 & 290.29 & 09 & 63005 & 17 & efe & & 10 & 3. & 4.10 & 1.1 & .91 & 127 \\
\hline 杞麓湖 & 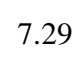 & & 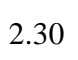 & 1.550 .43 & 20.6 & & & & & 3 & ( & 3.35 & 0.0820 \\
\hline & 2.09 & & 53 & 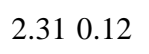 & & & & & & & 0.4 & 2.50 & 483 \\
\hline & 1 & & 55 & 0.700 .06 & & & & & 2.31 & 0 & 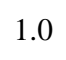 & 35 & 10 \\
\hline & & & & & & & & & & 0 & ( & 5 & \\
\hline & & & 40 & & & & & & & 73 & .5 & 43 & \\
\hline & 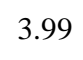 & & & & & & & & & & 5 & 1.54 & \\
\hline & 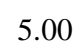 & & 6 & & & & & & & U & 0 & 19 & 99 \\
\hline & 168 & 2 & 75 & 0 & 1 & & & & 2.35 & 3.00 & 1 & 49 & 53 \\
\hline & & & & I & & & & & & & ( & & \\
\hline & 2 & & 52 & 50 & & & & & & 50 & 1.5 & 5 & ES \\
\hline & 2.0 & 0.16 & 90 & 3 & & & & 1 & 9.42 & 00 & 0.6 & 69 & 351 \\
\hline & & & & . & & & & & & & ( & 0 & \\
\hline & & 3.560 .72 & 3.58 & 90 & & & & 06 & & 40.27 & 0.3 & .10 & \\
\hline & 3 & 0.590 .10 & 7 & 320 & 25 & & & 0.16 & 1.10 & 2.07 & 4 & 9 & 11 \\
\hline & .99 & 3.440 .76 & 3.20 & 990. & & & & & & 27.73 & 0.4 & .92 & .050 \\
\hline & 5.88 & 2.990 .58 & 2.32 & 300.17 & 19.7 & 20.60 & 21.33 & 0.06 & 4.18 & 15.00 & 0.6 & 1.38 & 0.1128 \\
\hline & 13. & & 51 & 010 & & & & 0.02 & 14.18 & 29.13 & .5 & 59 & 0.1499 \\
\hline & 1.26 & 0.590 .09 & 07 & 550.04 & t & 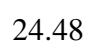 & 4.94 & -0.05 & 2.33 & 4.00 & 0.6 & 0.33 & 0.0021 \\
\hline
\end{tabular}

* Chl.a: 叶绿素a浓度, TSM: 总悬浮物浓度, SD: 透明度, TN: 总氮, TP: 总磷. 
型 2 统计参数 $R^{2}$ 和 $R M S E$ 均要显著高于模型 1 (ANOVA, $P<0.001$ ). 这主要是由于取自然对数进行线性拟合 时将很大的权重放到 $\mathrm{CDOM}$ 吸收非常低的长波红光部分，而在长波部分 $\mathrm{CDOM}$ 吸收由于比较低, 测定的 噪声和误差都比较大 ${ }^{[19]}$, 从而造成拟合的效果远远不如非线性拟合. 当然如果拟合的波段越窄并越集中 在短波部分, 模型 1 和模型2拟合的结果差别就越小. 如Helms等 ${ }^{[22]}$ 就曾报道在 $275-295 \mathrm{~nm}$ 以及 $350-400 \mathrm{~nm}$ 波段范围内模型 1 和 2 得到 $S$ 值差别小于 $1 \%$. 这里需要提醒的是许多研究在EXCEL和SPSS软件里拟合得 到的CDOM光谱斜率事实上是模型1的结果, 并非真正意义的非线性拟合的结果. 模型 3 增加了一个背景 选项主要是为了消除长波 $\mathrm{CDOM}$ 吸光度测定中的噪声以及由非CDOM吸收引起的吸收系数的波动. 相比 于CDOM吸收系数, 背景项 $K$ 值总体上比较小, 并且不同站点正负不一, 与CDOM吸收之间没有明显的某 种相关性(表2). 从 3 种模型拟合的 $S$ 值大小来看, 模型 3 的最大, 模型 2 次之, 模型 1 最小, 模型 3 相对于模型 1 光谱斜率 $S$ 均值增加 $6.2 \%$, 这与其他人的研究结果是非常一致 ${ }^{[19]}$. 如Stedmon等 ${ }^{[19]}$ 对丹麦沿海水域的研 究发现模型3得到300-650nm范围内S均值相比模型1增加了 $13 \%$.

表3 3种模型拟合CDOM光谱吸收

Tab.3 Comparison of statistical parameters and values of three models

\begin{tabular}{ccccc}
\hline & & 模型 1 & 模型 2 & 模型 3 \\
\hline$R^{2}$ & 范围 & $0.9319-0.9999$ & $0.9681-1.0000$ & $0.9781-1.0000$ \\
& 均值士标准差 & $0.9895 \pm 0.0166$ & $0.9971 \pm 0.0054$ & $0.9977 \pm 0.0040$ \\
$R M S E\left(\mathrm{~m}^{-1}\right)$ & 范围 & $0.032-0.567$ & $0.025-0.217$ & $0.025-0.206$ \\
& 均值士标准差 & $0.239 \pm 0.149$ & $0.097 \pm 0.057$ & $0.083 \pm 0.044$ \\
光谱斜率 $S\left(\mu \mathrm{m}^{-1}\right)$ & 范围 & $16.96-20.08$ & $16.08-28.75$ & $14.96-33.82$ \\
& 均值士标准差 & $20.98 \pm 3.15$ & $21.58 \pm 2.90$ & $22.28 \pm 3.72$ \\
\hline
\end{tabular}

\section{2 颗粒物吸收}

从颗粒物吸收变化来看, 不同湖泊间总颗粒物的光谱吸收系数差异很大, $a_{\mathrm{p}}(440)$ 的值在 0.09-5.78 $\mathrm{m}^{-1}$ 间变化(表 2), 均值为 $1.39 \pm 1.44 \mathrm{~m}^{-1}$, 其值远低于富营养化浅水湖泊太湖的值 ${ }^{[17]}$. 从曲线的形状来看大致 随波长的增加吸收系数逐渐降低(图 2), 非常类似非色素颗粒物吸收光谱, 除杞麓湖、听湖、星云湖 3 个 湖泊叶绿素 a 浓度非常高(表 2), 在 $675 \mathrm{~nm}$ 附近会出现明显吸收峰外, 其它湖泊在 $675 \mathrm{~nm}$ 附近要么没吸收 峰要么吸收峰非常弱, 说明总体上而言颗粒物吸收以非色素颗粒物为主. 叶绿素 a 浓度与颗粒物吸收系 数 $a_{\mathrm{p}}(440) 、 a_{\mathrm{p}}(675)$ 与统计相关分析发现, 发现它们之间均存在显著正相关, 但与 $a_{\mathrm{p}}(675)$ 的相关性明显要 高于 $a_{\mathrm{p}}(440)$ (表 4). 考虑到杞麓湖、听湖、星云湖 3 个湖泊叶绿素 a 浓度明显高于其它湖泊会影响相关性 检验, 将这 3 个湖剔除之后进行统计分析, 发现叶绿素 $\mathrm{a}$ 浓度与颗粒物吸收系数 $a_{\mathrm{p}}(440) 、 a_{\mathrm{p}}(675)$ 相关性 均明显下降, 并且叶绿素 $\mathrm{a}$ 浓度与 $a_{\mathrm{p}}(440)$ 已不存在显著正相关. 因而可以判断, 对于叶绿素 $\mathrm{a}$ 浓度非常高 的杞麓湖、听湖、星云湖浮游植物的吸收很大程度上决定了总颗粒物吸收, 但对于其它湖泊而言, 浮游 植物的只会在 $675 \mathrm{~nm}$ 附近吸收峰处对总颗粒物吸收有一定影响, 而在其它波段则影响较小, 整体上颗粒 物组成中浮游植物所占比例比较低, 主要以非色素颗粒物为主. 造成不同湖泊间总颗粒物吸收光谱存在 差异主要原因是不同湖泊中颗粒物的组成, 尤其是浮游植物和非色素颗粒物比重存在很大差别, 但是由 于未进行实验分离浮游植物和非色素颗粒物各自吸收系数, 因而无从确定它们各自所占的比例. 从颗粒 物吸收与悬浮物浓度之间的相关性来看, 它们之间存在极显著正相关, 并且杞麓湖、听湖、星云湖 3 个 湖泊是否剔除对其相关性影响不大，因而表 4 只给出全部湖泊的线性回归结果.

\subsection{CDOM 和颗粒物吸收对总吸收的贡献}

表 5 给出 CDOM 和颗粒物在 400-700nm 光合有效辐射(PAR)及 350-400nm 紫外辐射(UVR)波段积 分吸收系数、各自的贡献份额. 图 3 是各湖泊 CDOM 和颗粒物吸收对总吸收贡献率的光谱分布. CDOM 吸收对总吸收的贡献主要在 $600 \mathrm{~nm}$ 以下波长, $600 \mathrm{~nm}$ 以上其贡献基本上可以忽略不计, 随波长的降低贡 
表 4 颗粒物吸收与叶绿素 $\mathrm{a}$ 、悬浮物浓度之间的相关性

Tab.4 Linear correlations between total particulate matter absorption coefficients $a_{\mathrm{p}}(675)$, $a_{\mathrm{p}}(440)$ and Chl.a, TSM concentrations

\begin{tabular}{cccccc}
\hline & & & 关系式 & $R^{2}$ & $P$ \\
\hline \multirow{2}{*}{ Chl.a } & 全部湖泊 & $a_{\mathrm{p}}(675)$ & $a_{\mathrm{p}}(675)=0.006( \pm 0.001) C h l . \mathrm{a}+0.085( \pm 0.027)$ & 0.65 & $<0.001$ \\
& & $a_{\mathrm{p}}(440)$ & $a_{\mathrm{p}}(440)=0.018( \pm 0.008) C h l . \mathrm{a}+1.150( \pm 0.255)$ & 0.14 & $<0.05$ \\
& 剔除杞麓湖、 & $a_{\mathrm{p}}(675)$ & $a_{\mathrm{p}}(675)=0.017( \pm 0.005) C h l . \mathrm{a}+0.041( \pm 0.033)$ & 0.25 & $<0.01$ \\
& 听湖、星云湖 & $a_{\mathrm{p}}(440)$ & $a_{\mathrm{p}}(440)=0.113( \pm 0.061) C h l . \mathrm{a}+0.746( \pm 0.365)$ & 0.11 & $=0.07$ \\
\hline \multirow{2}{*}{$\mathrm{TSM}$} & \multirow{2}{*}{ 全部湖泊 } & $a_{\mathrm{p}}(675)$ & $a_{\mathrm{p}}(675)=0.011( \pm 0.002) \mathrm{TSM}-0.019( \pm 0.046)$ & 0.48 & $<0.001$ \\
& & $a_{\mathrm{p}}(440)$ & $a_{\mathrm{p}}(440)=0.075( \pm 0.011) \mathrm{TSM}+0.103( \pm 0.242)$ & 0.60 & $<0.001$ \\
\hline
\end{tabular}

献率逐渐增加, 尤其是在 $400 \mathrm{~nm}$ 以下的紫外波 段贡献率的增加尤为明显. 34 个湖泊平均而言, CDOM 在 PAR 和 UVR 波段的贡献率均值分别 为 $15.5 \% \pm 11.0 \% 、 46.8 \% \pm 21.3 \%$. UVR 波段的 贡献显著要大于 PAR 波段(ANOVA, $P<0.001$ ). 考虑到 $\mathrm{CDOM}$ 吸收随波长降低按近似指数函 数增长, 可以推断在更短的 UV-B 辐射波段 $\mathrm{CDOM}$ 吸收对总吸收的贡献将继续增加. 颗粒 物吸收对总吸收的贡献率总体上保持在一个较 高的水平, 最高值一般出现 500-550nm 附近, 随波长降低或增加其贡献率都有所下降, 但在 $675 \mathrm{~nm}$ 附近有颗粒物中浮游植物在此波段的特 征吸收峰, 相应的贡献率也存在一个峰值. 全 部湖泊平均而言, 颗粒物在 PAR 和 UVR 波段 的贡献率均值比较接近, 分别为 $51.1 \% \pm 16.9 \% 、 51.9 \% \pm 21.5 \%$. 34 个湖泊平均而 言, 在 350-700nm 绝大部分波段, 颗粒物吸收 的贡献率均要大于 CDOM, 仅在 $360 \mathrm{~nm}$ 之后 CDOM 吸收贡献大于颗粒物, 但可以预见的是 随波长继续降低, 到更短波的 UV-B 辐射, CDOM 吸收的贡献率会越来越大于颗粒物.

为了更好讨论湖泊间 CDOM 和颗粒物吸 收对总吸收的贡献率, 将全部 34 个湖泊以透 明度 $\mathrm{SD}=1.0$ 为界分为 2 类, $\mathrm{SD}<1.0$ 为浑浊型 湖泊, $\mathrm{SD} \geqslant 1.0$ 为清澈型湖泊(表 3 ). 对于浑浊 型湖泊而言, 颗粒物吸收对总吸收的贡献无论 是在 PAR 还是 UVR 波段都明显上升, 其均值 分别为 $60.9 \% \pm 14.6 \% 、 60.9 \% \pm 22.5 \%$. 对于这类
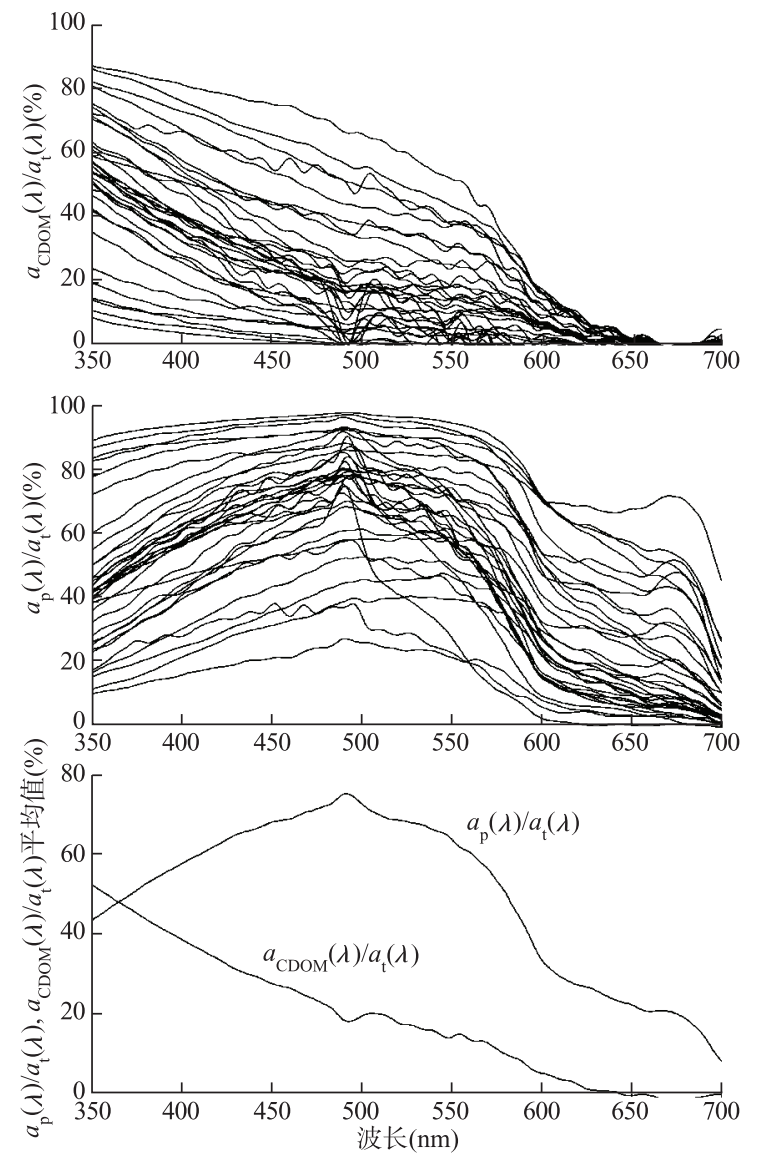

图 3 CDOM 和颗粒物吸收对总吸收的贡献率

Fig.3 Spectral variations of the contributions of CDOM and particulate matter to the total absorption 湖泊而言, 水体内悬浮物颗粒物浓度较高, 叶绿素 a 和总悬浮物浓度平均值分别为 $22.55 \pm 38.96 \mu \mathrm{g} / \mathrm{L}$ 和 $24.02 \pm 14.49 \mathrm{mg} / \mathrm{L}$, 分别为清澈型湖泊均值的 9.5 和 2.8 倍, 并且统计上浑浊型湖泊悬浮物浓度显著高于清 澈型湖泊(ANOVA, $P<0.001)$. 高浓度悬浮物颗粒物明显增加光的吸收和散射, 也会造成紫外辐射的衰减 增加 ${ }^{[8-9]}, U V-B$ 辐射影响的深度非常有限. 对于清澈型湖泊而言, CDOM吸收对总吸收的贡献则显著增加, PAR、UVR 波段贡献率的均值分别为 $19.6 \% \pm 11.0 \% 、 57.3 \% \pm 14.2 \%$ ，明显高于浑浊型湖泊的 $12.2 \% \pm 10.1 \%$ 、 
$38.5 \% \pm 22.5 \%$, 尤其是在 UVR 波段贡献率的增加尤为明显. 对于这类湖泊, 紫外辐射在湖内的衰减则很 大程度上取决于 CDOM 吸收. 尽管 CDOM 吸收系数 $a_{\mathrm{CDOM}}$ (320)的均值比浑浊型湖泊低(其均值分别为 $\left.3.96 \pm 4.02 \mathrm{~m}^{-1} 、 5.51 \pm 3.66 \mathrm{~m}^{-1}\right)$, 但由于水体内颗粒物浓度非常低, CDOM 对紫外辐射衰减的贡献则明显上 升. 并且对这类湖泊来说, 由于浮游植物、非藻类颗粒物和 CDOM 含量都非常低, 紫外辐射衰减比较小, UV-B 辐射能影响的深度则明显增加. 如根据经验模型计算得到此次采样李家凹 $320 \mathrm{~nm}$ 处 UV-B 辐射漫 射衰减系数为 $0.73 \mathrm{~m}^{-1}, \mathrm{UV}-\mathrm{B}$ 辐射可能影响的深度达 $6.32 \mathrm{~m}^{[18]}$. 又如 2006 年 10 月对抚仙湖的紫外辐射测 定显示, $320 \mathrm{~nm}$ 处 UV-B 辐射衰减系数为 $0.97 \mathrm{~m}^{-1}$, UV-B 辐射可能影响的深度达 $4.75 \mathrm{~m}$.

表 5 光合有效辐射和紫外辐射波段 CDOM、颗粒物吸收系数积分值及各自贡献*

Tab.5 The integral values of CDOM, particulate matter and the total absorption during PAR and UVR ranges, and their relative contributions

\begin{tabular}{|c|c|c|c|c|c|c|c|c|c|c|c|}
\hline & & \multicolumn{5}{|c|}{ PAR (400-700)波段 } & \multicolumn{5}{|c|}{ UVR (350-400)波段 } \\
\hline & & $a_{\mathrm{CDOM}}$ & $a_{\mathrm{p}}$ & $a_{\mathrm{t}}$ & $a_{\mathrm{CDOM}} / a_{\mathrm{t}}$ & $a_{\mathrm{p}} / a_{\mathrm{t}}$ & $a_{\mathrm{CDOM}}$ & $a_{\mathrm{p}}$ & $a_{\mathrm{t}}$ & $a_{\mathrm{CDOM}} / a_{\mathrm{t}}$ & $a_{\mathrm{p}} / a_{\mathrm{t}}$ \\
\hline \multirow{4}{*}{$\begin{array}{l}\text { 全部 } \\
\text { 湖泊 }\end{array}$} & 最小值 & 0.02 & 0.05 & 0.22 & 0.8 & 15.9 & 0.22 & 0.15 & 0.42 & 6.3 & 13.7 \\
\hline & 最大值 & 0.56 & 2.57 & 2.78 & 42.7 & 80.8 & 5.22 & 11.56 & 13.15 & 85.4 & 93.4 \\
\hline & 均值 & 0.19 & 0.65 & 0.99 & 15.5 & 51.1 & 1.80 & 2.44 & 4.27 & 46.8 & 51.9 \\
\hline & 标准差 & 0.16 & 0.67 & 0.70 & 11.0 & 16.9 & 1.49 & 2.84 & 3.25 & 21.3 & 21.5 \\
\hline \multirow{4}{*}{$\begin{array}{c}\mathrm{SD}< \\
1.0 \text { 浑浊 } \\
\text { 型湖泊 }\end{array}$} & 最小值 & 0.02 & 0.25 & 0.43 & 0.8 & 28.8 & 0.40 & 0.96 & 1.39 & 6.3 & 16.7 \\
\hline & 最大值 & 0.54 & 2.58 & 2.78 & 37.8 & 80.8 & 5.08 & 11.56 & 13.15 & 82.9 & 93.4 \\
\hline & 均值 & 0.21 & 0.98 & 1.35 & 12.2 & 60.9 & 2.03 & 3.69 & 5.74 & 38.5 & 60.9 \\
\hline & 标准差 & 0.16 & 0.73 & 0.73 & 10.1 & 14.6 & 1.40 & 3.23 & 3.16 & 22.5 & 22.5 \\
\hline \multirow{4}{*}{$\begin{array}{c}\mathrm{SD} \geq 1.0 \\
\text { 清澈型 } \\
\text { 湖泊 }\end{array}$} & 最小值 & 0.02 & 0.04 & 0.22 & 6.7 & 15.9 & 0.22 & 0.15 & 0.42 & 35.1 & 13.7 \\
\hline & 最大值 & 0.56 & 0.72 & 1.43 & 42.7 & 47.5 & 5.22 & 3.80 & 8.81 & 85.4 & 60.3 \\
\hline & 均值 & 0.16 & 0.22 & 0.54 & 19.6 & 38.7 & 1.52 & 0.86 & 2.41 & 57.3 & 40.5 \\
\hline & 标准差 & 0.17 & 0.16 & 0.31 & 11.0 & 9.9 & 1.16 & 0.88 & 2.33 & 14.2 & 13.7 \\
\hline
\end{tabular}

$* a_{\mathrm{CDOM}}, a_{\mathrm{p}}, a_{\mathrm{t}}$ 单位为 $\mathrm{m}^{-1} ; a_{\mathrm{CDOM}} / a_{\mathrm{t}}, a_{\mathrm{p}} / a_{\mathrm{t}}$ 单位为 $\%$.

从 CDOM 和颗粒物吸收与透明度的关系也可以发现, 不同类型湖泊主导水下光衰减的因子不一样. 对 于浑浊型湖泊而言, 颗粒物吸收 $a_{\mathrm{p}}(355)$ 与透明度存在显著幂函数负相关 $\left(R^{2}=0.32, P<0.05\right)$, 而 CDOM 吸收 $a_{\mathrm{CDOM}}(355)$ 与透明度没有显著性关系, 说明浑浊型湖泊颗粒物吸收和散射主导水下光场衰减. 但对于清澈 型湖泊而言, $\mathrm{CDOM}$ 吸收 $a_{\mathrm{CDOM}}(355)$ 与透明度存在显著幂函数负相关 $\left(R^{2}=0.45, P<0.01\right)$, 尽管颗粒物吸收 $a_{\mathrm{p}}(355)$ 与透明度也存在幂函数负相关 $\left(R^{2}=0.32, P<0.05\right)$, 但其相关性要明显低于 CDOM 吸收. 因此对于这 类湖泊而言, $\mathrm{CDOM}$ 的吸收对光衰减的影响是主导性, 并且考虑到 $\mathrm{CDOM}$ 吸收随波长降低近似按指数函数增 加, 在更短的 UV-B 辐射波段这种主导性会更明显, 世界其它清澈型高原湖泊中的研究结果也能很好的佐证 本研究 ${ }^{[4,7,14]}$.

\section{3 结论}

(1)不同湖泊间 CDOM 吸收差异明显, $a_{\mathrm{CDOM}}(320)$ 的变化范围和均值分别为 $0.65-14.05 \mathrm{~m}^{-1}$ 、 $4.83 \pm 3.85 \mathrm{~m}^{-1}$. CDOM 吸收与湖泊营养水平相关, $a_{\mathrm{CDOM}}(320)$ 与水体总氮存在显著正相关. 增加背景常数项 的指数函数模型能最好较好模拟 CDOM 光谱吸收.

(2)整体而言, 颗粒物吸收呈现非色素颗粒物吸收光谱特征, 随波长增加逐渐降低. 但在叶绿素 a 浓 度非常高的杞麓湖、听湖、星云湖, 颗粒物吸收系数在 $675 \mathrm{~nm}$ 附近存在一个明显吸收峰外. 悬浮物浓度 能很好解释颗粒物吸收系数变化, 将杞麓湖、听湖、星云湖剔除之后, 叶绿素 a 浓度与颗粒物吸收相关性 明显下降, 叶绿素 $\mathrm{a}$ 的解释率有限.

(3)CDOM 对总吸收的贡献集中在短波尤其是 400nm 以下的紫外波段, 其在紫外波段的贡献明显要 
大于光合有效辐射波段. 对 $\mathrm{SD}<1.0$ 的浑浊型湖泊，无论紫外波段和光合有效辐射波段，颗粒物吸收对 总吸收的贡献均要大于 $C D O M$; 而对于透明度 $\mathrm{SD} \geq 1.0$ 的清澈型湖泊, CDOM 吸收在紫外波段的贡献要大 于颗粒物, 其吸收很大程度上决定了紫外辐射的影响深度.

致谢：野外采样得到李春海、董旭辉、唐红渠的帮助，在此表示诚挚的谢意.

\section{4 参考文献}

[1] Madronich S, McKenzie RE, Björn LO et al. Changes in biologically active ultraviolet radiation reaching the Earth’s surface. $J$ Photochem Photobiol B:Biology, 1998, 46: 5-19.

[2] 周秀骥, 李维亮, 陈隆勋等. 青藏高原地区大气臭氧变化的研究. 气象学报, 2004, 62: 513-527.

[3] Morris DP, Zagarese HE, Williamson CE et al. The attenuation of solar UV radiation in lakes and the role of dissolved organic carbon. Limnol Oceanogr, 1995, 40: 1381-1391.

[4] Laurion I, Ventura M, Catalan J et al. Attenuation of ultraviolet radiation in mountain lakes: Factors controlling the among-and within-lake variability. Limnol Oceanogr, 2000, 45: 1274-1288.

[5] Scully NM, Lean DRS. The attenuation of UV radiation in temperate lakes. Arch Hydrobiol Ergeb Limnol, 1994, 43: 135-144.

[6] Laurion I, Vincent WF, Lean DRS. Underwater ultraviolet radiation: Development of spectral models for northern high latitude lakes. Photochem Photobiol, 1997, 65: 107-114.

[7] Morris DP, Hargreaves BP. The role of photochemical degradation of dissolved organic carbon in regulating the UV transparency of three lakes on the Pocono Plateau. Limnol Oceanogr, 1997, 42: 239-249.

[8] Smith REH, Furgal JA, Charlton MN et al. Attenuation of ultraviolet radiation in a large lake with low dissolved organic matter concentrations. Can J Fish Aquat Sci, 1999, 56: 1351-1361.

[9] Belzilel C, Vincent WF, Kumagai M. Contribution of absorption and scattering to the attenuation of UV and photosynthetically available radiation in Lake Biwa. Limnol Oceanogr, 2002, 47: 95-107.

[10] De Lange HJ. The attenuation of ultraviolet and visible radiation in Dutch inland waters. Aquatic Ecol, 2000, 34: 215-226.

[11] Vincent WF, Kumagai M, Belzile C et al. Effects of seston on ultraviolet attenuation in Lake Biwa. Limnology, 2001, 2: 179-184.

[12] 王苏民, 窦鸿身. 中国湖泊志. 北京: 科学出版社, 1998.

[13] Caldwell MM, Robberecht R, Billings WD. A steep latitudinal gradient of solar ultraviolet-B radiation in the arctic-alpine lift zone. Ecology, 1980, 61: 600-611.

[14] Sommaruga R. The role of solar UV radiation in the ecosystem of alpine lakes. J Photochem Photobio B: Biol, 2001,62 : 35-42.

[15] Williamson CE. Effects of UV radiation on freshwater ecosystems. Intern J Environmental Studies, 1996, 51: $245-256$.

[16] Zhang YL, Zhang EL, Liu ML et al. Variation of chromophoric dissolved organic matter and possible attenuation depth of ultraviolet radiation in Yunnan Plateau lakes. Limnology, 2007, 8: 311-319.

[17] Zhang YL, Zhang B, Wang X et al. A study of absorption characteristics of chromophoric dissolved organic matter and particles in Lake Taihu, China. Hydrobiologia, 2007, 592: 105-120.

[18] Markager W, Vincent WF. Spectral light attenuation and absorption of UV and blue light in natural waters. Limnol Oceanogr, 2000, 45: 642-650.

[19] Stedmon CA, Markager S, Kaas H. Optical properties and signatures of chromophoric dissolved organic matter (CDOM) in Danish coastal waters. Estuar Coast Shelf S, 2000, 51: 267-278.

[20] Zhang YL, Qin BQ, Zhang L et al. Spectral absorption and fluorescence of chromophoric dissolved organic matter in shallow lakes in the middle and lower reaches of the Yangtze River. J Freshwater Ecol, 2005, 20(3): 451-459.

[21] Hayakawa K, Sakamoto M, Kumagai M et al. Fluorescence spectroscopic characterization of dissolved organic matter in the waters of Lake Fuxian and adjacent rivers in Yunnan, China. Limnology, 2004, 5: 155-163.

[22] Helms JR, Stubbins A, Ritchie JD et al. Absorption spectral slopes and slope ratios as indicators of molecular weight, source, and photobleaching of chromophoric dissolved organic matter. Limnol Oceanogr, 2008, 53: 955-969. 